\title{
Schwierige Ursachensuche
}

\section{Warum muss das Kind ständig husten?}

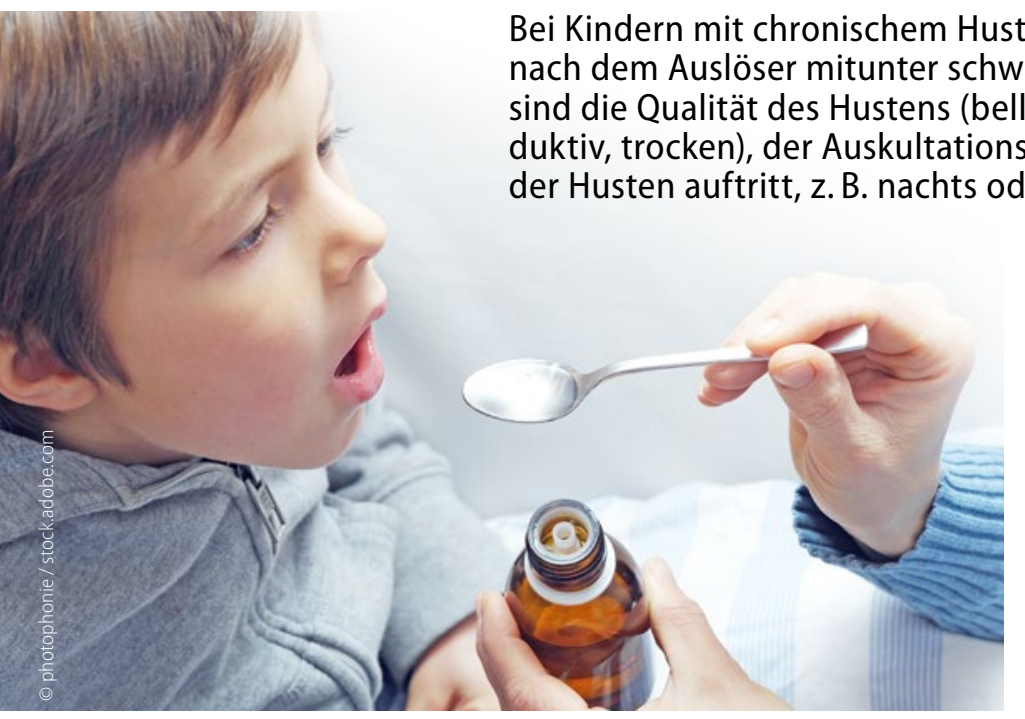

Bei habituellem Husten helfen Medikamente in der Regel nicht.

Zur Abklärung eines chronischen, länger als vier Wochen anhaltenden Hustens beim Kind sind Anamnese und Auskultation zunächst die wichtigsten Instrumente. Ist ein Giemen oder Stridor zu hören, deutet dies auf eine Obstruktion hin, sagte Prof. Dr. Jürgen Seidenberg aus Oldenburg. Rasselgeräusche können durch eine Bronchiolitis oder Alveolitis ausgelöst werden, eine Bronchophonie ist ein möglicher Hinweis auf eine Pneumonie und Seitendifferenz für einen Erguss, eine Atelektase oder Überblähung.

\section{Husten bei Fremdkörperaspiration}

Die Auskultation kann auch den Verdacht einer Fremdkörperaspiration verstärken, die zu 70\% mit Husten einhergeht. Bei etwa der Hälfte der Kinder mit Fremdkörperaspiration sind die Atemgeräusche abgeschwächt oder es ist beim Atmen ein Pfeifen zu hören, so Seidenberg.

Auch die Qualität des Hustens liefert wichtige Hinweise auf die Ursache. Bei Kleinkindern liegt häufig ein feuchtschleimiger Husten vor, der meist nicht pneumoniae oder Moraxella catarrhalis. Betroffen sind nach Erfahrung von Seidenberg meist Kinder unter 2 Jahren. Für eine Symptomfreiheit sind oft zwei, selten mehr antibiotische Zyklen nötig.

\section{Denken Sie auch an Mukoviszidose}

Bei Kindern mit feucht-schleimigem Husten sollte aber auch an Immundefekte, Mukoviszidose und chronische Sinusitis aufgrund vergrößerter Adenoide, auch „upper airway cough syndrom“ (UACS) genannt, gedacht werden. Zu den Therapieoptionen bei UACS zählen nasale Steroide, bei Verdacht auf eine allergische Anamnese auch Antihistaminika und Leukotrienantagonisten.

Auch ein Stakkatohusten wird häufig durch eine Infektion ausgelöst, etwa durch Chlamydien oder durch respiratorische Synzytial-Viren (RSV). Ist am Ende der Ausatmung ein Keuchen zu hören, sollte auch an Pertussis gedacht werden, erklärte Seidenberg. Er erinnerte an eine britische Studie bei 170 Schulkindern mit persistierendem Husten. Bei mehr als zwei Drittel der Patienten zeigten sich serologische Hinweise auf eine akute Pertussisimpfung, obwohl 86\% der Kinder eine Grundimmunsierung erhalten hatten [1].
Bei trockenem Husten, vor allem unter körperlicher Belastung und nachts, besteht dringender Verdacht auf Asthma, sagte Seidenberg. Als Risikofaktoren für ein meist allergisches Asthma im Schulkindalter nannte er u.a. positive Familienanamnese, atopisches Ekzem im Säuglingsalter, Giemen im infektfreien Intervall und vermehrte Eosinophile. Ist die Diagnose unklar, kann auch ein Therapieversuch mit inhalativem Kortikosteroid (plus lang wirksamen Bronchodilatatoren) über zwei bis drei Wochen erfolgen, berichtete Seidenberg. Höre der Husten auf, liege Asthma bzw. eine asthmaähnliche Erkrankung vor, sonst müsse weitergefahndet werden.

Bei anhaltendem produktivem Husten kann ein diagnostischer Therapieversuch mit Antibiotika erfolgen. Sei der Husten in zwei bis drei Wochen vorbei, lag eine protrahierte Bronchitis vor, sonst sei eine weitere Diagnostik erforderlich.

\section{Verdacht auf habituellen Husten}

Bei unklarem röhrendem Husten sollte an einen habituellen Husten gedacht werden. Laut Seidenberg ist er v. a. bei Jungen im Alter von sieben bis zu zehn Jahren häufig. Typischerweise leiden die Kinder v.a. in Gegenwart der Eltern oder der Lehrer an extrem störendem Husten ohne somatische Ursache. Die Symptome können monatelang anhalten. Hustenmedikamente sind in der Regel nicht wirksam.

Als weitere Ursachen eines chronischen Hustens nannte Seidenberg eine Bronchitis fibroplastica, die zu produktivem Husten mit Fibrinfäden führt, Husten in Verbindung mit Thoraxschmerzen, die auf eine Arrhythmie, Embolie, eine Pneumonie oder eine Pleuritis hindeuten können, sowie angeborene Herzfehler mit begleitenden Gefäßanomalien oder Herzinsuffizienz.

Roland Fath

112. Jahrestagung der Deutschen Gesellschaft für Kinderund Jugendmedizin, Hamburg, September 2016

1. Harnden A, BMJ 2006; 333(7560):174-7 